\title{
DESIGN AND SYNTHESIS OF HETEROCYCLIC ANALOGS OF ENEDIYNE ANTIBIOTICS
}

\author{
I. Balova, N.A. Danilkina, A.I. Govdi \\ Institute of Chemistry, Saint Petersburg University, \\ 199034, Russia, Saint Petersburg, Universitetskaya nab., 7/9
}

DOI: 10.19163/MedChemRussia2021-2021-55

E-mail: i.balova@spbu.ru

Enediyne antibiotics are natural compounds with a strong DNA damaging effect due to ability the (Z)-3-en-1,5-diyne fragment included in a strained cycle, to undergo Bergman cyclization at human body temperature, and the resulting 1,4-phenylene biradicals provide DNA breaks. Along with high cytotoxicity, natural enediyne antibiotics have a very low stability after isolation, as well as low selectivity in a relation to cancerous and healthy cells, which limits their use. We have designed and synthesized series of enediyne analogues fused with heterocycles and also containing a heteroatoms in 10-membered enediyne cycles [1-6]. The annulation of various heterocycles with enediyne makes it possible to influence the rate of cycloaromatization with the formation of a key diradical intermediate also use the heterocycle as a site to modify the enediyne molecule and increase its affinity for DNA. The investigation of binding of the obtained macrocyclic enediyne systems to DNA using calculation and experimental methods, as well as their DNA-damaging activity on model plasmids, cytotoxicity, and antiproliferative activity on various lines of tumor cell will also be discussed.

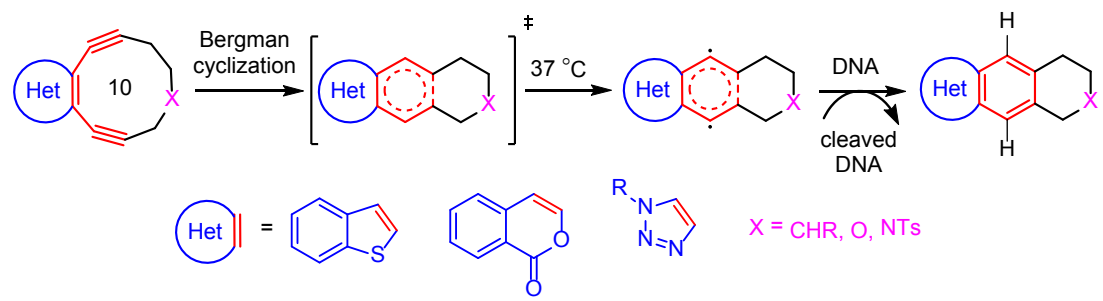

This work was supported by Russian Foundation for Basic Research (project No 21-13-00218).

\section{References}

[1] Danilkina N.A., Kulyashova A.E., Khlebnikov A.F.; Bräse, S., Balova, I.A. J. Org. Chem., 2014, 79 (19) 9018-9045.

[2] Danilkina, N.A.; Gurskaya, L.Yu.; Vasilyev, A.V.; Balova, I.A. Eur.J. Org. Chem, 2016, 4, 739-747.

[3] Lyapunova AG., Danilkina N. A,. Khlebnikov A. F., Köberle B., Bräse S., Balova I. A. Eur. J. Org. Chem, 2016, 28, 4842-4851.

[4] Lyapunova, AG., Danilkina, N. A., Rumyantsev A. M., Khlebnikov A. F., Chislov M.V., Starova G.L., Sambuk E.V., Govdi A.I., Bräse S., Balova I. A. J. Org. Chem,. 2018, 83, 2788-2801.

[5] Govdi A.I., Danilkina N.A., Ponomarev A.V., Balova I. A. J. Org. Chem,. 2019, 84, 1925-1940

[6] Danilkina N.A., D’yachenko A., Govdi A.I., Khlebnikov A.F., Kornyakov I., Bräse S., Balova I. A., J. Org. Chem,. 2020, 85, 14, 9001-9014. 